J. Indones. Math. Soc.

Special Edition (2011), pp. 59-69.

\title{
ON THE SUPER EDGE-MAGIC DEFICIENCY AND $\alpha$-VALUATIONS OF GRAPHS
}

\author{
Rikio IChishima ${ }^{1}$ AND Akito Oshima ${ }^{2}$ \\ ${ }^{11}$ College of Humanities and Sciences, Nihon University, 3-25-40 \\ Sakurajousui Setagaya-ku, Tokyo 156-8550, Japan, \\ ichishim@chs.nihon-u.ac.jp \\ Department of Mathematical Information Science, Faculty of Science, Tokyo \\ University of Science, 1-3 Kagurazaka Shinjuku-ku, Tokyo 162-8601, Japan, \\ akito_o@rs.kagu.tus.ac.jp
}

\begin{abstract}
A graph $G$ is called super edge-magic if there exists a bijective function $f: V(G) \cup E(G) \rightarrow\{1,2, \ldots,|V(G)|+|E(G)|\}$ such that $f(u)+f(v)+f(u v)$ is a constant for each $u v \in E(G)$ and $f(V(G))=\{1,2, \ldots,|V(G)|\}$. The super edge-magic deficiency, $\mu_{s}(G)$, of a graph $G$ is defined as the smallest nonnegative integer $n$ with the property that the graph $G \cup n K_{1}$ is super edge-magic or $+\infty$ if there exists no such integer $n$. In this paper, we prove that if $G$ is a graph without isolated vertices that has an $\alpha$-valuation, then $\mu_{s}(G) \leq|E(G)|-|V(G)|+1$. This leads to $\mu_{s}(G)=|E(G)|-|V(G)|+1$ if $G$ has the additional property that $G$ is not sequential. Also, we provide necessary and sufficient conditions for the disjoint union of isomorphic complete bipartite graphs to have an $\alpha$-valuation. Moreover, we present several results on the super edge-magic deficiency of the same class of graphs. Based on these, we propose some open problems and a new conjecture.
\end{abstract}

Key words: Super edge-magic labeling, super edge-magic deficiency, sequential labeling, sequential number, $\alpha$-valuation.

2000 Mathematics Subject Classification: Primary 05C78.

Received: 09-08-2011, revised: 09-09-2011, accepted: 04-12-2012. 